\title{
Synthesis of Novel Fluorinated Hexa-peri-hexabenzocoronenes
}

\author{
Olivier F. Aebischer, ${ }^{\mathrm{a}}$ Patrick Tondo, ${ }^{\mathrm{a}}$ Bassam Alameddine, ${ }^{\mathrm{b}}$ Titus A. Jenny*a \\ a Chemistry Department, University of Fribourg, 9 chemin du Musée, 1700 Fribourg, Switzerland \\ Fax +41(26)3009739; E-mail: titus.jenny@unifr.ch \\ b Chemistry Department, University of Balamand, P.O. Box 100, Tripoli, Lebanon \\ Received 5 May 2006; revised 24 May 2006
}

\begin{abstract}
The synthesis of several perfluoroalkylated hexabenzocoronene derivatives is described. The substituents range from linear semiperfluoroalkylated chains to branched perfluoroalkylated chains. Novel strategies were applied to overcome the low solubility and the low reactivity of such chains.
\end{abstract}

Key words: coronenes, tolanes, perfluoroalkyl substituents, crosscoupling, cyclodehydrogenation

Polycondensed aromatic hydrocarbons (PAHs) represent a widely investigated class of aromatic compounds and have been extensively studied over the past decades. ${ }^{1}$ Hexa-peri-hexabenzocoronene (HBC), an all-benzoid PAH containing thirteen fused benzene rings, possesses high thermal and chemical stability. It is also known to self-assemble into highly ordered architectures, ${ }^{2}$ rendering the $\mathrm{HBC}$ moiety a candidate of high potential for applications in plastic electronics. Many functionalised HBCs, either with extended conjugated structures, or with alkyl groups on their periphery, have been prepared, ${ }^{3}$ but only few bearing perfluoroalkylated side chains have been reported. ${ }^{4,5}$ Those highly fluorinated side chains are expected to clad the HBC molecule with a Teflon-like mantle that reduces the tendency for any lateral aggregation. ${ }^{5}$

We report herein the synthesis of three new HBC derivatives bearing linear and branched fluorinated side chains (Figure 1).

The best strategy for the synthesis of symmetrically substituted hexa-peri-hexabenzocoronene ${ }^{6}$ derivatives $\mathbf{1}$ in-

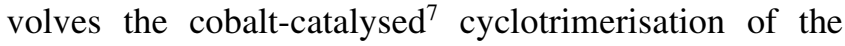
corresponding disubstituted tolane derivatives $\mathbf{3}$, yielding hexaphenylbenzene moieties $\mathbf{2}$, which are subsequently cyclodehydrogenated, under the Kovacic conditions, ${ }^{8}$ in which the iron(III) chloride reagent acts as both Lewis acid and oxidant (Scheme 1). The latter conditions, however, prevent the preparation of HBC derivatives in which perfluorinated side chains are directly attached to the core. An alkyl spacer of at least two carbons, or an ether or thioether linkage is required to reduce the electron-withdrawing effect of the perfluorinated side chain to an acceptable level for the mild iron(III) chloride reagent to be successful. ${ }^{5}$ The main task remains, therefore, in the preparation of the symmetrically substituted tolane derivatives $\mathbf{3}$ (Scheme 1). Nonetheless, the drastic change in both reactivity and solubility of highly fluorinated derivatives did not allow the application of the conditions already reported in the literature ${ }^{9}$ to the preparation of alkylated tolane derivatives. The Kumada cross-coupling of a bis-brominated tolane with perfluoroalkyl Grignard intermediates, for example, requires up to one week of reaction time and yields the desired tolane only in unreliable and at best very moderate yields, ${ }^{5}$ whereas for regular alkyl Grignard species the reaction works without difficulties in a short time and in acceptable yields. ${ }^{9}$

A better way to prepare perfluoroalkylated tolane derivatives consists therefore of synthesising first the halogenated precursors 5a-c (Schemes 2 and 3), as these compounds are highly soluble in common organic sol-<smiles>[R]c1ccc(C#Cc2ccc([R])cc2)cc1</smiles>

Scheme 1 Retrosynthesis ${ }^{3}$ showing strategic bond disconnection for the preparation of hexa-peri-hexabenzocoronenes

SYNTHESIS 2006, No. 17, pp 2891-2896

Advanced online publication: 15.08 .2006

DOI: 10.1055/s-2006-942550; Art ID: Z09406SS

(c) Georg Thieme Verlag Stuttgart · New York 


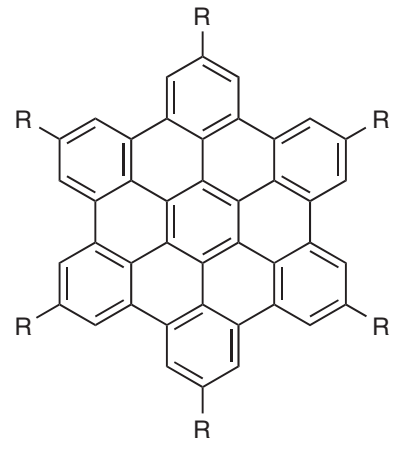

1a: $\mathrm{R}=\left(\mathrm{CH}_{2}\right)_{2}\left(\mathrm{CF}_{2}\right)_{6} \mathrm{~F}$ 1b: $\mathrm{R}=\left(\mathrm{CH}_{2}\right)_{4}\left(\mathrm{CF}_{2}\right)_{6} \mathrm{~F}$ 1c: $\mathrm{R}=\left(\mathrm{CH}_{2}\right)_{3} \mathrm{CH}\left[\mathrm{CH}_{2}\left(\mathrm{CF}_{2}\right)_{6} \mathrm{~F}\right]_{2}$

Figure 1 Hexa-peri-hexabenzocoronene target molecules

vents, allowing for their preparation in high purity. The tolane derivatives 3a-c are then obtained in a one-pot Sonogashira reaction, ${ }^{10}$ as white, sparingly soluble compounds (Scheme 4).

Precursor 5a is already described in the literature, ${ }^{11}$ whereas precursors $\mathbf{5 b}$ and $\mathbf{5 c}$ are, to our knowledge, new derivatives and represent very interesting fluorinated building blocks.

For the synthesis of compound $\mathbf{5 b}$ (Scheme 2), 1-bromo4-but-3-enylbenzene (4) was first coupled with perfluorohexyl iodide in a radical reaction. Sodium metabisulfite was added to temper the reaction and to reduce the amount of side products. ${ }^{12}$ Of all tested dehalogenation methods, the use of lithium aluminum hydride was best for removal of the iodine of the side chain of $\mathbf{6}$. Under these condi- tions, only the iodine of the side chain of $\mathbf{6}$ reacts, without debromination of the aromatic core occurring. Finally, bromobenzene 7 was converted into the more reactive iodobenzene $\mathbf{5 b}$ by a halogen-exchange ${ }^{13}$ reaction. Compound $\mathbf{5 b}$ was isolated in $40 \%$ overall yield for the three steps and in high purity after silica gel column chromatography.

The preparation of precursor 5c started with the Kumada cross-coupling of the known fluorinated iodoalkene $\mathbf{8}^{14}$ with allylmagnesium bromide. The coupling only succeeded when the Grignard derivative was added very slowly (over several hours) at $0{ }^{\circ} \mathrm{C}$; otherwise, many side products formed. The Heck reaction between fluorinated alkene 9 and benzenediazonium $\mathbf{1 0}$ proved to be a challenge. Coupling was attempted in the presence of palladium(II) acetate and methanol, but no product formed at all. Fortunately, the coupling was finally achieved when palladium(II) acetate, calcium carbonate, and a methanoltetrahydrofuran mixture $(3: 1)$ was used at $50{ }^{\circ} \mathrm{C}$. Purification of the crude product by column chromatography over silica gel afforded styrene derivative $\mathbf{1 1}$ as a colourless oil in moderate yield. Hydrogenation was achieved under mild conditions with the use of a rhodium-carbon catalyst, ${ }^{11}$ to prevent the dehalogenation of the aromatic core. Finally, compound 5c was isolated as a colourless liquid in $34 \%$ isolated overall yield.

The most efficient way to convert an aromatic halide into a tolane derivative involves a Sonogashira coupling. Many different conditions are proposed in literature, but
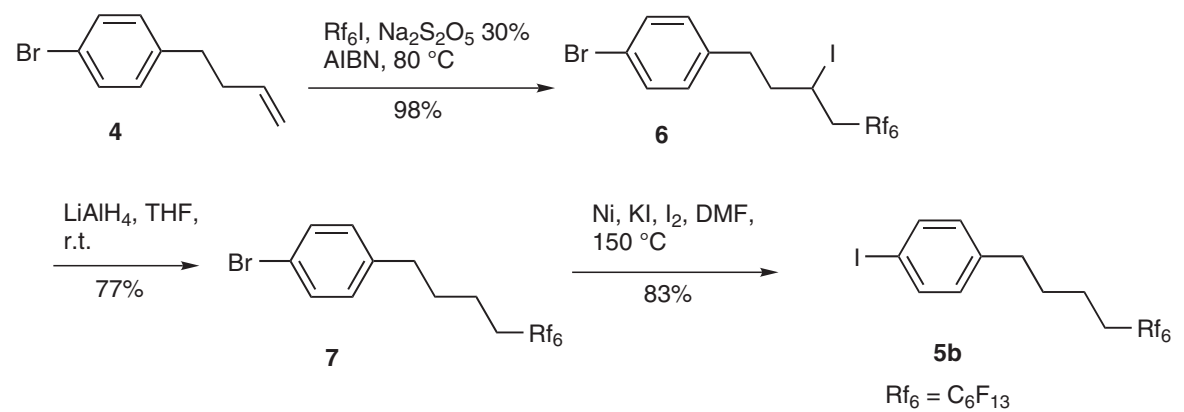

Scheme 2 Three-step synthesis of fluoroalkyl-substituted iodobenzene precursor $\mathbf{5 b}$

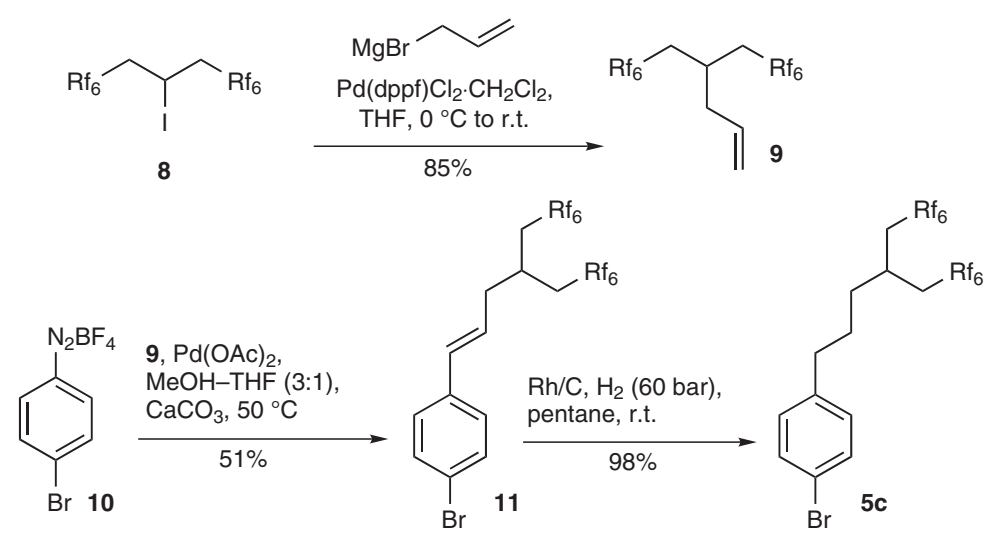

Scheme 3 Synthesis of fluoroalkyl-substituted bromobenzene precursor $\mathbf{5 c}$ by Kumada and Heck coupling 


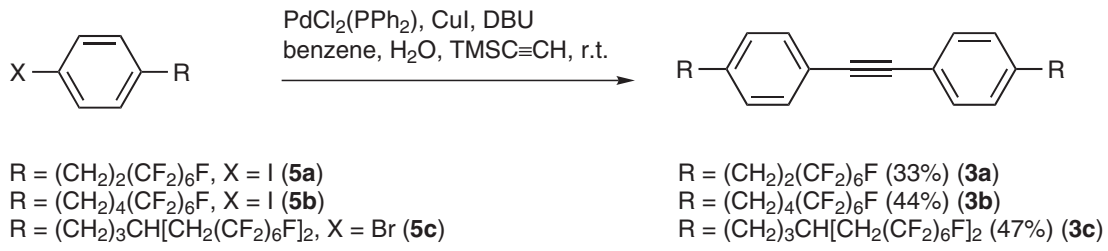

Scheme 4 Sonogashira one-pot coupling of fluoroalkylated halobenzenes with (trimethylsilyl)acetylene to yield tolane derivatives 3a-3c

most of them proceed stepwise, starting with the coupling of the appropriate halobenzene precursor with (trimethylsilyl)acetylene, followed by deprotection of the trimethylsilyl group and, finally, by coupling this product with the second halobenzene. In our case, however, this approach failed at the deprotection stage for solubility reasons. Fortunately, two methods were found to perform the coupling in a one-pot reaction. The first ${ }^{15}$ uses a constant stream of acetylene passing through the reaction mixture at $80^{\circ} \mathrm{C}$. As the obtained yields were generally low, a second method was applied, in which (trimethylsilyl)acetylene ${ }^{10}$ was used instead. Despite the problems encountered in the stepwise procedure, the highly fluorinated precursors $\mathbf{5 a}-$ c yielded the tolane derivatives $\mathbf{3 a}-\mathbf{c}$, although in moderate yields owing to their difficult purification: linear perfluoroalkylated tolane derivatives $\mathbf{3 a}$ and $\mathbf{3 b}$ are only slightly soluble in common organic solvents, and were purified by recrystallisation and filtration over basic aluminum oxide. Nevertheless, the method avoids the formation of homocoupled products, normally occurring in one-pot procedures owing to the very low solubility of the alkynylated intermediates. It has to be noted that even minor admixtures of homocoupled products totally prevent the subsequent cyclotrimerisation reaction from occurring and that, in the case of the perfluorinated side chains, these byproducts cannot be removed by chromatography.

Cyclotrimerisation $^{7}$ of all tolane derivatives 3a-c (Scheme 5) was achieved in high yield, provided that they were of high purity. Finally, cyclodehydrogenation ${ }^{8}$ was performed in the presence of a large excess of iron(III) chloride (typically 20- to 60-fold) in nitromethane, and under a constant stream of argon to remove the formed hy- drogen chloride, to yield the $\mathrm{HBC}$ derivatives $\mathbf{1 a}-\mathbf{c}$ in fair to good yields (45-70\%) (Scheme 5). Final purification was by recrystallisation from benzotrifluoride, hexafluorobenzene, and diethyl ether.

In conclusion, three new symmetrically substituted hexaperi-hexabenzocoronenes bearing linear or branched partially fluorinated alkyl chains in their periphery were prepared and characterised. These HBC derivatives 1a-c are potential candidates in plastic electronics, while their precursors are new, interesting partially fluorinated building blocks.

All reagents and solvents were purchased from commercial sources and used without further purification. THF and $\mathrm{CH}_{2} \mathrm{Cl}_{2}$ were dried and deoxygenated by the Grubbs method. ${ }^{16}$ All reactions were performed under inert atmosphere $\left(\mathrm{N}_{2}\right.$ or $\left.\mathrm{Ar}\right) .{ }^{1} \mathrm{H}$ NMR and ${ }^{13} \mathrm{C}$ NMR spectra were recorded on a Bruker DPX 360 or a Bruker DRX 500 spectrometer, using $\mathrm{CDCl}_{3}$ or hexafluorobenzene as solvent. Chemical shifts $(\delta)$ are reported in ppm, and coupling constants $(J)$ are given in Hz. Mass spectra were recorded on a Finnigan Thermo Quest GC/MS Voyager spectrometer or a Bruker 4.7 T BioAPEX II FT/ICR spectrometer. Analytical TLC was performed on Merck Kieselgel 60 F254 pre-coated glass plates and visualised by spraying with a $\mathrm{KMnO}_{4}$ soln.

\section{1-Bromo-4-(5,5,6,6,7,7,8,8,9,9,10,10,10-tridecafluoro-3-iodode- cyl)benzene (6)}

Perfluorohexyl iodide (2.6 g, $5.86 \mathrm{mmol}$ ) was syringed into a roundbottomed flask equipped with a reflux condenser and heated to $50{ }^{\circ} \mathrm{C}$. After injection of a $30 \%$ aq soln of $\mathrm{Na}_{2} \mathrm{~S}_{2} \mathrm{O}_{5}(1 \mathrm{~mL})$, followed by the addition of AIBN $(19 \mathrm{mg}, 0.11 \mathrm{mmol})$ and $4(1.2 \mathrm{~g}, 5.68$ $\mathrm{mmol})$, the mixture was heated to $80^{\circ} \mathrm{C}$ for $4 \mathrm{~h} . \mathrm{H}_{2} \mathrm{O}(20 \mathrm{~mL})$ was then added and the product was extracted with $\mathrm{CH}_{2} \mathrm{Cl}_{2}(3 \times 50 \mathrm{~mL})$. The combined organic phases were washed with $\mathrm{H}_{2} \mathrm{O}(3 \times 50 \mathrm{~mL})$, dried $\left(\mathrm{Na}_{2} \mathrm{SO}_{4}\right)$, filtered, and evaporated; this afforded the title compound as a colourless oil.
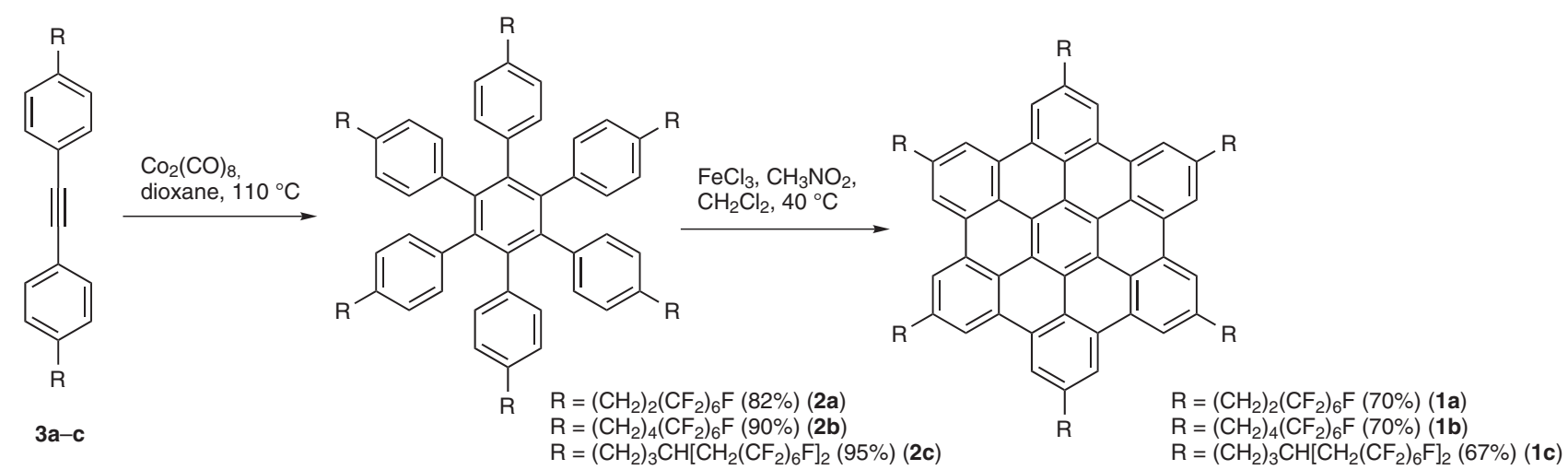

Scheme 5 Trimerisation and cyclodehydrogenation of tolanes yielding the desired hexa-peri-hexabenzocoronene derivatives 1a-c 
Yield: $3.67 \mathrm{~g}$ (98\%); $R_{f}=0.63$ (pentane).

${ }^{1} \mathrm{H}$ NMR $\left(360 \mathrm{MHz}, \mathrm{CDCl}_{3}\right): \delta=7.42(\mathrm{~d}, J=8.2 \mathrm{~Hz}, 2 \mathrm{H}, \mathrm{Ar}), 7.19$ (d, $J=8.2 \mathrm{~Hz}, 2 \mathrm{H}, \mathrm{Ar}), 4.23$ (tt, $J=8.6,5.0 \mathrm{~Hz}, 1 \mathrm{H}, \mathrm{CHI}), 2.48$ $3.04\left(\mathrm{~m}, 4 \mathrm{H}, \mathrm{CH}_{2}\right), 2.00-2.17\left(\mathrm{~m}, 2 \mathrm{H}, \mathrm{CH}_{2}\right)$.

${ }^{13} \mathrm{C}$ NMR (90.55 MHz, $\left.\mathrm{CDCl}_{3}\right): \delta=138.78,131.70,130.24$, 107.56-121.07 (m, Rf $), 120.21,41.69,41.73(\mathrm{t}, J=21.1 \mathrm{~Hz})$, 41.39, 35.13, 19.71 .

MS (EI, $70 \mathrm{eV}): \mathrm{m} / \mathrm{z}(\%)=656.9(5)\left[\mathrm{M}^{+}\right], 529.6(13), 170.8(100)$, 116.9 (44), 89.9 (75).

\section{1-Bromo-4-(5,5,6,6,7,7,8,8,9,9,10,10,10-tridecafluorodecyl)ben- zene (7)}

To a slurry of LAH (574 mg, $15.1 \mathrm{mmol})$ in THF ( $5 \mathrm{~mL}$ ) was added $6(5.0 \mathrm{~g}, 7.56 \mathrm{mmol})$ in THF $(10 \mathrm{~mL})$. After $3 \mathrm{~h}$ of reaction at r.t., the mixture was quenched by the addition of $\mathrm{H}_{2} \mathrm{O}(5 \mathrm{~mL}), 1 \mathrm{M}$ $\mathrm{NaOH}(5 \mathrm{~mL})$, and $\mathrm{H}_{2} \mathrm{O}$ again $(15 \mathrm{~mL})$. The suspension was filtered and washed exhaustively with $\mathrm{Et}_{2} \mathrm{O}(5 \times 100 \mathrm{~mL})$. The organic phase was separated, washed with $\mathrm{H}_{2} \mathrm{O}(3 \times 30 \mathrm{~mL})$, dried $\left(\mathrm{Na}_{2} \mathrm{SO}_{4}\right)$, filtered, and evaporated. Purification by column chromatography (silica gel, pentane) afforded the title compound as a colourless oil.

Yield: $3.4 \mathrm{~g}(77 \%) ; R_{f}=0.63$ (pentane).

${ }^{1} \mathrm{H}$ NMR $\left(360 \mathrm{MHz}, \mathrm{CDCl}_{3}\right): \delta=7.41(\mathrm{~d}, J=8.2 \mathrm{~Hz}, 2 \mathrm{H}, \mathrm{Ar}), 7.05$ (d, $J=8.2 \mathrm{~Hz}, 2 \mathrm{H}, \mathrm{Ar}), 2.61\left(\mathrm{t}, J=7.0 \mathrm{~Hz}, 2 \mathrm{H}, \mathrm{CH}_{2}\right), 2.01-2.15$ (m, $\left.2 \mathrm{H}, \mathrm{CH}_{2}\right), 1.61-1.73\left(\mathrm{~m}, 4 \mathrm{H}, \mathrm{CH}_{2}\right)$.

${ }^{13} \mathrm{C} \mathrm{NMR}\left(90.55 \mathrm{MHz}, \mathrm{CDCl}_{3}\right): \delta=140.55,131.49,130.08,119.73$, 108.07-121.99 (m, $\mathrm{Rf}_{6}$ ), 34.94, 30.69, 30.69 (t, $\left.J=22.5 \mathrm{~Hz}\right), 19.75$ (t, $J=3.6 \mathrm{~Hz})$.

MS (EI, $70 \mathrm{eV}): m / z(\%)=530.7(28)\left[\mathrm{M}^{+}\right], 170.8(100), 91(68)$.

\section{1-Iodo-4-(5,5,6,6,7,7,8,8,9,9,10,10,10-tridecafluorodecyl)ben- zene (5b)}

To a mixture of $\mathrm{Ni}(54.8 \mathrm{mg}, 0.94 \mathrm{mmol}), \mathrm{KI}$ (62 mg, $0.37 \mathrm{mmol})$, $\mathrm{I}_{2}(2.3 \mathrm{mg}, 9.3 \mu \mathrm{mol})$, and deoxygenated $7(0.1 \mathrm{~g}, 0.19 \mathrm{mmol})$ was added DMF $(0.5 \mathrm{~mL})$. The resulting suspension was heated to $150{ }^{\circ} \mathrm{C}$ for $27 \mathrm{~h}$. The crude reaction mixture was allowed to cool to r.t., before $3 \%$ aq $\mathrm{HCl}(10 \mathrm{~mL})$ was added. The resulting mixture was extracted with pentane $(3 \times 20 \mathrm{~mL})$. The combined organic layers were dried $\left(\mathrm{Na}_{2} \mathrm{SO}_{4}\right)$ and filtered, and all volatiles were removed. This yielded a brown liquid, which was filtered over a plug of silica gel under reduced pressure with pentane as eluent; this afforded the title compound as a colourless liquid.

Yield: $90 \mathrm{mg}$ (83\%); $R_{f}=0.65$ (pentane).

${ }^{1} \mathrm{H}$ NMR $\left(360 \mathrm{MHz}, \mathrm{CDCl}_{3}\right): \delta=7.61(\mathrm{~d}, J=8.2 \mathrm{~Hz}, 2 \mathrm{H}, \mathrm{Ar}), 6.93$ (d, $J=8.2 \mathrm{~Hz}, 2 \mathrm{H}, \mathrm{Ar}), 2.60$ (t, $J=7.04 \mathrm{~Hz}, 2 \mathrm{H}, \mathrm{CH}_{2}$ ), 2.00-2.15 (m, $\left.2 \mathrm{H}, \mathrm{CH}_{2}\right), 1.61-1.71\left(\mathrm{~m}, 4 \mathrm{H}, \mathrm{CH}_{2}\right)$.

${ }^{13} \mathrm{C} \mathrm{NMR}\left(90.55 \mathrm{MHz}, \mathrm{CDCl}_{3}\right): \delta=141.22,137.47,130.44,90.98$, $107.65-121.48\left(\mathrm{~m}, \mathrm{Rf}_{6}\right), 35.03,30.68(\mathrm{t}, J=22.5 \mathrm{~Hz}), 30.65,19.76$ $\left(\mathrm{t},{ }^{3} J_{\mathrm{CF}}=3.64 \mathrm{~Hz}\right)$.

MS (EI, $70 \mathrm{eV}): m / z(\%)=578.8(12)\left[\mathrm{M}^{+}\right], 216.9(100), 89.9(21)$.

\section{8-Allyl-1,1,1,2,2,3,3,4,4,5,5,6,6,10,10,11,11,12,12,13,13,14,14,-} $15,15,15$-hexacosafluoropentadecane $(9)$

An oven-dried Schlenk flask was charged with $\left[\mathrm{Pd}(\mathrm{dppf}) \mathrm{Cl}_{2}\right]$ $\mathrm{CH}_{2} \mathrm{Cl}_{2}(253 \mathrm{mg}, 0.31 \mathrm{mmol})$ and deoxygenated $8(5 \mathrm{~g}, 6.2 \mathrm{mmol})$, diluted with THF $(20 \mathrm{~mL})$. The mixture was cooled to $0{ }^{\circ} \mathrm{C}$ in an ice- $\mathrm{NaCl}$ bath. Then $1 \mathrm{M}$ allylmagnesium bromide in $\mathrm{Et}_{2} \mathrm{O}(9.3 \mathrm{~mL}$, $9.3 \mathrm{mmol}$ ) was syringed in slowly $(2 \mathrm{~h})$, and this yielded a yellow soln. The ice bath was removed after the addition was completed and the mixture (turning green) was stirred for $12 \mathrm{~h}$ at r.t. before being quenched with $\mathrm{MeOH}(10 \mathrm{~mL})$. All volatiles were removed under reduced pressure; a brown suspension remained, which was filtered over a silica-gel plug under reduced pressure with pentane as solvent. After evaporation of pentane, the title compound was obtained as a colourless oil.

Yield: $3.79 \mathrm{~g}(85 \%) ; R_{f}=0.86$ (pentane- $\left.\mathrm{Et}_{2} \mathrm{O}, 4: 1\right)$.

${ }^{1} \mathrm{H}$ NMR $\left(360 \mathrm{MHz}, \mathrm{CDCl}_{3}\right.$ ): $\delta=5.72(\mathrm{ddt}, J=17.3,10.0,6.8 \mathrm{~Hz}$, $1 \mathrm{H}, \mathrm{CH}), 5.20\left(\mathrm{~d}, J=17.3 \mathrm{~Hz}, 1 \mathrm{H}, \mathrm{CH}_{2}\right), 5.15(\mathrm{~d}, J=10.0 \mathrm{~Hz}, 1$ $\mathrm{H}, \mathrm{CH}_{2}$ ), 2.55 (sept, $\left.J=6.8 \mathrm{~Hz}, 1 \mathrm{H}, \mathrm{CH}\right), 2.34(\mathrm{t}, J=6.8 \mathrm{~Hz}, 2 \mathrm{H}$, $\left.\mathrm{CH}_{2}\right), 2.13-2.27\left(\mathrm{~m}, 4 \mathrm{H}, \mathrm{CH}_{2}\right)$.

${ }^{13} \mathrm{C}$ NMR $\left(90.55 \mathrm{MHz}, \mathrm{CDCl}_{3}\right): \delta=133.5,119.32,105.25-122.33$ $\left(\mathrm{m}, \mathrm{Rf}_{6}\right), 38.80,33.60(\mathrm{t}, J=21.1 \mathrm{~Hz}), 24.94$.

MS (EI, $70 \mathrm{eV}): m / z(\%)=721.2(2)\left[\mathrm{M}^{+}\right], 378.3(100)$.

1-Bromo-4-[6,6,7,7,8,8,9,9,10,10,11,11,11-tridecafluoro-4$(2,2,3,3,4,4,5,5,6,6,7,7,7$-tridecafluoroheptyl)undec-1-enyl]benzene (11)

To a suspension of $\mathbf{1 0}(5.4 \mathrm{~g}, 20 \mathrm{mmol}), \mathrm{Pd}(\mathrm{OAc})_{2}(90 \mathrm{mg}, 0.4$ $\mathrm{mmol})$, and $\mathrm{CaCO}_{3}(2 \mathrm{~g}, 20 \mathrm{mmol})$ in $\mathrm{MeOH}(12 \mathrm{~mL})$ was added 9 $(14.4 \mathrm{~g}, 20 \mathrm{mmol})$ in THF $(36 \mathrm{~mL})$. The mixture was heated to $50{ }^{\circ} \mathrm{C}$ and stirred for $44 \mathrm{~h}$. Removal of all volatiles yielded a crude brown solid, which was suspended in pentane and filtered over a plug of silica gel under reduced pressure with pentane as eluent. Evaporation of the pentane afforded the crude reaction product, which was purified by column chromatography (silica gel, pentane). Evaporation of pentane from the combined fractions yielded the title compound as a colourless oil.

Yield: $8.98 \mathrm{~g}(51 \%) ; R_{f}=0.55$ (pentane).

${ }^{1} \mathrm{H}$ NMR $\left(360 \mathrm{MHz}, \mathrm{CDCl}_{3}\right): \delta=7.45(\mathrm{~d}, J=8.2 \mathrm{~Hz}, 2 \mathrm{H}, \mathrm{Ar}), 7.22$ $(\mathrm{d}, J=8.2 \mathrm{~Hz}, 2 \mathrm{H}, \mathrm{Ar}), 6.43(\mathrm{~d}, J=15.9 \mathrm{~Hz}, 1 \mathrm{H}, \mathrm{CH}), 6.06(\mathrm{dt}$, $J=15.9,7.3 \mathrm{~Hz}, 1 \mathrm{H}, \mathrm{CH}), 2.63(\mathrm{sept}, J=5.9 \mathrm{~Hz}, 1 \mathrm{H}, \mathrm{CH}), 2.49(\mathrm{t}$, $\left.J=7.3 \mathrm{~Hz}, 2 \mathrm{H}, \mathrm{CH}_{2}\right), 2.17-2.30\left(\mathrm{~m}, 4 \mathrm{H}, \mathrm{CH}_{2}\right)$.

${ }^{13} \mathrm{C} \mathrm{NMR}\left(90.55 \mathrm{MHz}, \mathrm{CDCl}_{3}\right): \delta=135.61,133.29,131.73,127.69$, $125.65,121.45,107.81-118.87\left(\mathrm{~m}, \mathrm{Rf}_{6}\right), 37.96,33.76$ (t, $J=20.8$ $\mathrm{Hz}), 25.48$.

MS (EI, $70 \mathrm{eV}): m / z(\%)=873.98(85)\left[\mathrm{M}^{+}\right], 461.04(12), 198.03$ (100).

\section{1-Bromo-4-[6,6,7,7,8,8,9,9,10,10,11,11,11-tridecafluoro-4- $(2,2,3,3,4,4,5,5,6,6,7,7,7$-tridecafluoroheptyl)undecyl]benzene (5c)}

Bromobenzene 11 (9.6 g, $10.98 \mathrm{mmol})$ was dissolved in pentane (90 $\mathrm{mL}$ ) and placed in a $250-\mathrm{mL}$ autoclave. The $\mathrm{Rh} / \mathrm{C}(450 \mathrm{mg}, 0.44$ mmol) catalyst was added, and the autoclave was purged with $\mathrm{H}_{2}$ $(2 \times 60$ bar $)$. The mixture was stirred at r.t. under $\mathrm{H}_{2}(50 \mathrm{bar})$ for 24 h. Afterwards, the catalyst was removed by filtration over a silicagel plug under reduced pressure with pentane as solvent. Removing all volatiles yielded the title compound as a colourless oil.

Yield: $9.4 \mathrm{~g}(98 \%) ; R_{f}=0.70$ (pentane).

${ }^{1} \mathrm{H}$ NMR $\left(360 \mathrm{MHz}, \mathrm{CDCl}_{3}\right): \delta=7.41(\mathrm{~d}, J=8.2 \mathrm{~Hz}, 2 \mathrm{H}, \mathrm{Ar}), 7.05$ (d, $J=8.2 \mathrm{~Hz}, 2 \mathrm{H}, \mathrm{Ar}$ ), 2.59 (t, $J=6.5 \mathrm{~Hz}, 2 \mathrm{H}, \mathrm{CH}_{2}$ ), 2.44 (sept, $J=5.5 \mathrm{~Hz}, 1 \mathrm{H}, \mathrm{CH}), 2.07-2.24\left(\mathrm{~m}, 4 \mathrm{H}, \mathrm{CH}_{2}\right), 1.58-1.65(\mathrm{~m}, 4 \mathrm{H}$, $\mathrm{CH}_{2}$ ).

${ }^{13} \mathrm{C} \mathrm{NMR}\left(90.55 \mathrm{MHz}, \mathrm{CDCl}_{3}\right): \delta=140.52,131.51,130.05,119.80$, $35.02,34.27,34.22(\mathrm{t}, J=21.1 \mathrm{~Hz}), 27.78,25.40$.

MS (EI, $70 \mathrm{eV}): m / z(\%)=875.99(100)\left[\mathrm{M}^{+}\right]$.

\section{1,1'-Ethyne-1,2-diylbis[4-(3,3,4,4,5,5,6,6,7,7,8,8,8-tridecafluo-} rooctyl)benzene] (3a)

$\mathrm{CuI}(18 \mathrm{mg}, 99 \mu \mathrm{mol})$ and $\left[\mathrm{PdCl}_{2}\left(\mathrm{PPh}_{3}\right)_{2}\right](40 \mathrm{mg}, 59.6 \mu \mathrm{mol})$ were suspended in an oven-dried Schlenk flask in freshly distilled benzene $(2.5 \mathrm{~mL})$. Iodobenzene $\mathbf{5 a}(0.5 \mathrm{~g}, 0.99 \mathrm{mmol})$ was dissolved in benzene $(2.5 \mathrm{~mL})$ before being added to the reaction vessel followed by the injection of DBU $(0.88 \mathrm{~mL}, 5.94 \mathrm{mmol}) . \mathrm{H}_{2} \mathrm{O}(7 \mu \mathrm{L}$, $40 \mu \mathrm{mol})$ and (trimethylsilyl)acetylene $(66 \mu \mathrm{L}, 0.47 \mu \mathrm{mol})$ were added simultaneously by two syringes. The Schlenk flask was then 
protected from ambient light and heated to $80^{\circ} \mathrm{C}$. After $72 \mathrm{~h}$ of reaction, the black reaction mixture was allowed to cool to r.t. before $\mathrm{Et}_{2} \mathrm{O}(50 \mathrm{ml})$ was added. The crude mixture was extracted with $\mathrm{H}_{2} \mathrm{O}$ $(2 \times 50 \mathrm{~mL}), 10 \% \mathrm{HCl}(50 \mathrm{~mL})$, brine $(50 \mathrm{~mL})$ and $\mathrm{H}_{2} \mathrm{O}(50 \mathrm{~mL})$. The combined organic fractions were dried $\left(\mathrm{Na}_{2} \mathrm{SO}_{4}\right)$, filtered, and evaporated. This yielded a brown solid which was dissolved in $\mathrm{Et}_{2} \mathrm{O}$-pentane $(9: 1 ; 400 \mathrm{~mL})$ and filtered over a plug of basic aluminium oxide under reduced pressure. After evaporation of all volatiles, a yellow solid was obtained, which was further purified by recrystallisation from pentane- $\mathrm{Et}_{2} \mathrm{O}-\mathrm{EtOH}$; this yielded the title compound as an off-white solid.

Yield: $143 \mathrm{mg}(33 \%) ; R_{f}=0.46$ (pentane).

${ }^{1} \mathrm{H} \mathrm{NMR}\left(360 \mathrm{MHz}, \mathrm{CDCl}_{3}\right): \delta=7.49(\mathrm{~d}, J=8.1 \mathrm{~Hz}, 4 \mathrm{H}, \mathrm{Ar}), 7.20$ (d, $J=8.1 \mathrm{~Hz}, 4 \mathrm{H}, \mathrm{Ar}), 2.93\left(\mathrm{~m}, J=8.4 \mathrm{~Hz}, 4 \mathrm{H}, \mathrm{CH}_{2}\right), 2.23-2.44$ (m, $4 \mathrm{H}, \mathrm{CH}_{2}$ ).

${ }^{13} \mathrm{C}$ NMR (90.55 MHz, $\left.\mathrm{CDCl}_{3}\right): \delta=139.38,131.98,128.35$, 121.73, 108.38-119.99 (m, $\left.\mathrm{Rf}_{6}\right), 89.07,32.68(\mathrm{t}, J=22.1 \mathrm{~Hz})$, 26.40 .

MS (EI, $70 \mathrm{eV}): \mathrm{m} / \mathrm{z}(\%)=870.08(100)\left[\mathrm{M}^{+}\right], 481.27(20)$.

\section{1,1'-Ethyne-1,2-diylbis[4-(5,5,6,6,7,7,8,8,9,9,10,10,10-trideca- fluorodecyl)benzene] (3b)}

As described for compound 3a, iodobenzene 5b (9.9 g, 17.12 $\mathrm{mmol}$ ) reacted with (trimethylsilyl)acetylene $(1.32 \mathrm{~mL}, 9.08 \mathrm{mmol})$ in the presence of $\mathrm{CuI}(326 \mathrm{mg}, 1.71 \mathrm{mmol}),\left[\mathrm{PdCl}_{2}\left(\mathrm{PPh}_{3}\right)_{2}\right](721$ $\mathrm{mg}, 1.03 \mathrm{mmol})$, DBU (15.35 mL, $103 \mathrm{mmol})$, THF (80 mL), and $\mathrm{H}_{2} \mathrm{O}(123 \mu \mathrm{L}, 6.85 \mathrm{mmol})$.

Yield: $3.5 \mathrm{~g}(44 \%) ; R_{f}=0.95$ (pentane-EtOAc, 4:1).

${ }^{1} \mathrm{H}$ NMR $\left(500 \mathrm{MHz}, \mathrm{CDCl}_{3}\right): \delta=7.45(\mathrm{~d}, J=8.1 \mathrm{~Hz}, 4 \mathrm{H}, \mathrm{Ar}), 7.15$ $(\mathrm{d}, J=8.1 \mathrm{~Hz}, 4 \mathrm{H}, \mathrm{Ar}), 2.67\left(\mathrm{t}, J=7.5 \mathrm{~Hz}, 4 \mathrm{H}, \mathrm{CH}_{2}\right), 2.05-2.14$ (m, $\left.4 \mathrm{H}, \mathrm{CH}_{2}\right), 1.68-1.76\left(\mathrm{~m}, 8 \mathrm{H}, \mathrm{CH}_{2}\right)$.

${ }^{13} \mathrm{C}$ NMR $\left(125.77 \mathrm{MHz}, \mathrm{CDCl}_{3}\right): \delta=141.89,131.65,128.37$, $121.00,88.96,105.63-121.06\left(\mathrm{~m}, \mathrm{Rf}_{6}\right), 35.45,30.72(\mathrm{t}, J=22.1$ $\mathrm{Hz},), 30.62,19.79$.

MS (EI, $70 \mathrm{eV}): m / z(\%)=928.0(15)\left[\mathrm{M}^{+}\right], 565.9(100)$.

1,1'-Ethyne-1,2-diylbis $\{4-[6,6,7,7,8,8,9,9,10,10,11,11,11$-tridecafluoro-4-(2,2,3,3,4,4,5,5,6,6,7,7,7-tridecafluoroheptyl)undecyl]benzene\} (3c)

As described for compound 3a, bromobenzene $\mathbf{5 c}(2.8 \mathrm{~g}, 3.2 \mathrm{mmol})$ reacted with (trimethylsilyl)acetylene $(0.22 \mathrm{~mL}, 1.5 \mathrm{mmol})$ in the presence of $\mathrm{CuI}(60.8 \mathrm{mg}, 0.32 \mathrm{mmol}), \mathrm{PdCl}_{2}\left(\mathrm{PPh}_{3}\right)_{2}(134 \mathrm{mg}, 0.19$ $\mathrm{mmol})$, DBU $(2.7 \mathrm{~mL}, 19 \mathrm{mmol})$, benzene $(15 \mathrm{~mL})$, and $\mathrm{H}_{2} \mathrm{O}(23$ $\mu \mathrm{L}, 1.3 \mathrm{mmol})$.

Yield: $1.2 \mathrm{~g}(47 \%) ; R_{f}=0.15$ (pentane).

${ }^{1} \mathrm{H} \mathrm{NMR}\left(360 \mathrm{MHz}, \mathrm{CDCl}_{3}\right): \delta=7.46(\mathrm{~d}, J=8.2 \mathrm{~Hz}, 4 \mathrm{H}, \mathrm{Ar}), 7.15$ $(\mathrm{d}, J=8.2 \mathrm{~Hz}, 4 \mathrm{H}, \mathrm{Ar}), 2.65\left(\mathrm{t}, J=6.8 \mathrm{~Hz}, 4 \mathrm{H}, \mathrm{CH}_{2}\right), 2.45$ (sept, $J=5.9 \mathrm{~Hz}, 2 \mathrm{H}, \mathrm{CH}), 2.07-2.24\left(\mathrm{~m}, 8 \mathrm{H}, \mathrm{CH}_{2}\right), 1.58-1.65(\mathrm{~m}, 8 \mathrm{H}$, $\mathrm{CH}_{2}$ ).

${ }^{13} \mathrm{C}$ NMR (90.55 MHz, $\mathrm{CDCl}_{3}$ ): $\delta=141.83,131.66,128.35,121.06$, $108.05-120.83\left(\mathrm{~m}, \mathrm{Rf}_{6}\right), 88.95,35.52,34.30,34.24(\mathrm{t}, J=21.1 \mathrm{~Hz})$, 27.66, 25.40.

MALDI-ICR-MS (DCTB): $m / z$ calcd for $\mathrm{C}_{50} \mathrm{H}_{30} \mathrm{~F}_{52}: 1618.15$; found: $1618.15 ; \mathrm{m} / \mathrm{z}(\%)=1618.15(100)\left[\mathrm{M}^{+}\right], 1387.08(18)$, $922.10(15)$

\section{Hexakis[4-(3,3,4,4,5,5,6,6,7,7,8,8,8-tridecafluorooctyl)phe-} nyl]benzene (2a)

Tolane 3a $(0.1 \mathrm{~g}, 115 \mu \mathrm{mol})$ was dissolved in dioxane $(20 \mathrm{~mL})$. $\mathrm{Co}_{2}(\mathrm{CO})_{8}(2.4 \mathrm{mg}, 6.9 \mu \mathrm{mol})$ was added before the reaction mixture was heated to $110^{\circ} \mathrm{C}$. After $48 \mathrm{~h}$, the mixture was cooled to r.t. and all volatiles were removed, yielding a brown solid. Filtration through a plug of silica gel under reduced pressure with $\mathrm{Et}_{2} \mathrm{O}$ as sol- vent removed all traces of the catalyst and yielded a yellow solid which was recrystallised from $\mathrm{Et}_{2} \mathrm{O}-\mathrm{EtOH}$; this gave the title compound as a white solid.

Yield: $82 \mathrm{mg}$ (82\%); $R_{f}=0.85$ (pentane-EtOAc, 9:1).

${ }^{1} \mathrm{H}$ NMR $\left(360 \mathrm{MHz}, \mathrm{CDCl}_{3}\right): \delta=6.73(\mathrm{~d}, J=8.1 \mathrm{~Hz}, 12 \mathrm{H}, \mathrm{Ar}), 6.70$ $(\mathrm{d}, J=8.1 \mathrm{~Hz}, 12 \mathrm{H}, \mathrm{Ar}), 2.68(\mathrm{t}, J=7.9 \mathrm{~Hz}, 12 \mathrm{H}), 2.07-2.21(\mathrm{~m}$, $12 \mathrm{H})$.

${ }^{13} \mathrm{C} \mathrm{NMR}\left(90.55 \mathrm{MHz}, \mathrm{CDCl}_{3}\right): \delta=140.06,138.90,135.98,131.65$, 126.52, 109.25-120.15 (m, Rf $\left.)_{6}\right), 32.54(\mathrm{t}, J=22.1 \mathrm{~Hz}), 25.88$ (t, $J=4.0 \mathrm{~Hz}$ ).

MALDI-ICR-MS (DCTB): $m / z\left[\mathrm{M}^{+}\right](\%)$ calcd for $\mathrm{C}_{90} \mathrm{H}_{48} \mathrm{~F}_{78}$ : 2610.25; found: 2610.22 (100).

\section{Hexakis[4-(5,5,6,6,7,7,8,8,9,9,10,10,10-tridecafluorodecyl)phe-} nyl]benzene (2b)

As described for compound $\mathbf{2 a}$, tolane $\mathbf{3 b}(2.8 \mathrm{~g}, 3.02 \mathrm{mmol})$ was converted into the title compound in the presence of $\mathrm{Co}_{2}(\mathrm{CO})_{8}$ $(62.16 \mathrm{mg}, 0.18 \mathrm{mmol})$ and dioxane $(180 \mathrm{~mL})$.

Yield: $2.52 \mathrm{~g}$ (90\%); $R_{f}=0.19$ (pentane-EtOAc, 9:1).

${ }^{1} \mathrm{H} \mathrm{NMR}\left(360 \mathrm{MHz}, \mathrm{CDCl}_{3}\right): \delta=6.68(\mathrm{~d}, J=7.9 \mathrm{~Hz}, 12 \mathrm{H}, \mathrm{Ar}), 6.62$ $(\mathrm{d}, J=7.9 \mathrm{~Hz}, 12 \mathrm{H}, \mathrm{Ar}), 2.39\left(\mathrm{t}, J=7.0 \mathrm{~Hz}, 12 \mathrm{H}, \mathrm{CH}_{2}\right), 1.89-2.04$ $\left(\mathrm{m}, 12 \mathrm{H}, \mathrm{CH}_{2}\right), 1.46-1.53\left(\mathrm{~m}, 12 \mathrm{H}, \mathrm{CH}_{2}\right), 1.34-1.45(\mathrm{~m}, 12 \mathrm{H}$, $\mathrm{CH}_{2}$ ).

${ }^{13} \mathrm{C} \mathrm{NMR}\left(90.55 \mathrm{MHz}, \mathrm{CDCl}_{3}\right): \delta=140.19,138.53,137.85,131.58$, $126.45,108.07-121.19\left(\mathrm{~m}, \mathrm{Rf}_{6}\right), 34.62,30.55(\mathrm{t}, J=22.5 \mathrm{~Hz})$, $30.38,18.96$.

MALDI-ICR-MS (DCTB): $\mathrm{m} / \mathrm{z}\left[\mathrm{M}^{+}\right](\%)$ calcd for $\mathrm{C}_{102} \mathrm{H}_{72} \mathrm{~F}_{78}$ : 2778.44; found: 2778.45 (92).

\section{Hexakis $\{4-[6,6,7,7,8,8,9,9,10,10,11,11,11$-tridecafluoro-4- $(2,2,3,3,4,4,5,5,6,6,7,7,7$-tridecafluoroheptyl)undecyl]phe- nyl\}benzene (2c)}

As described for compound $2 \mathbf{a}$, tolane $\mathbf{3 c}(1.1 \mathrm{~g}, 0.67 \mathrm{mmol})$ was converted into the title compound in the presence of $\mathrm{Co}_{2}(\mathrm{CO})_{8}(18.6$ $\mathrm{mg}, 43 \mu \mathrm{mol})$ and dioxane $(20 \mathrm{~mL})$.

Yield: $1.05 \mathrm{~g}(95 \%) ; R_{f}=0.90$ (pentane).

${ }^{1} \mathrm{H} \mathrm{NMR}\left(500 \mathrm{MHz}, \mathrm{CDCl}_{3}\right): \delta=6.69(\mathrm{~d}, J=8.2 \mathrm{~Hz}, 12 \mathrm{H}, \mathrm{Ar}), 6.60$ $(\mathrm{d}, J=8.2 \mathrm{~Hz}, 12 \mathrm{H}, \mathrm{Ar}), 2.35(\mathrm{~m}, 6 \mathrm{H}, \mathrm{CH}), 2.34\left(\mathrm{~m}, 12 \mathrm{H}, \mathrm{CH}_{2}\right)$, 1.99-2.19 (m, $\left.24 \mathrm{H}, \mathrm{CH}_{2}\right), 1.35-1.46\left(\mathrm{~m}, 24 \mathrm{H}, \mathrm{CH}_{2}\right)$.

${ }^{13} \mathrm{C}$ NMR $\left(125.77 \mathrm{MHz}, \mathrm{CDCl}_{3}\right): \delta=140.16,138.59,137.81$, $131.55,126.33,110.56-120.60\left(\mathrm{~m}, \mathrm{Rf}_{6}\right), 34.73,34.11(\mathrm{t}, J=21.1$ $\mathrm{Hz}), 33.78,27.36,25.19$.

MALDI-ICR-MS (DCTB): $\mathrm{m} / z\left[\mathrm{M}^{+}\right](\%)$ calcd for $\mathrm{C}_{150} \mathrm{H}_{90} \mathrm{~F}_{156}$ : 4854.46; found: 4854.45 (48).

\section{2,5,8,11,14,17-Hexakis $(3,3,4,4,5,5,6,6,7,7,8,8,8$-tridecafluoro-} octyl)hexabenzo[bc,ef,hi,kl,no,qr]coronene (1a)

Hexaphenylbenzene 2a $(0.2 \mathrm{~g}, 76.6 \mu \mathrm{mol})$ was dissolved in dry $\mathrm{CH}_{2} \mathrm{Cl}_{2}(10 \mathrm{~mL})$. At the same time, a soln of $\mathrm{FeCl}_{3}(7.45 \mathrm{mg}, 4.59$ mmol) was prepared in $\mathrm{MeNO}_{2}(4 \mathrm{~mL})$. The $\mathrm{FeCl}_{3}$ soln was added drop-by-drop by syringe to the mixture at $45^{\circ} \mathrm{C}$. Argon was bubbled through the mixture through a Teflon capillary during all the reaction time. After $27 \mathrm{~h}$ of reaction, the mixture was quenched by the addition of $\mathrm{MeOH}(100 \mathrm{~mL})$. The black precipitate was collected by suction filtration over Millipore and was suspended in different common organic solvents $\left(\mathrm{CH}_{2} \mathrm{Cl}_{2}, \mathrm{MeOH}, \mathrm{Et}_{2} \mathrm{O}\right.$, and pentane). The formed suspensions were each time treated in an ultrasonic bath for $30 \mathrm{~min}$, followed by refluxing for $1 \mathrm{~h}$. After $2 \mathrm{~h}$ of cooling in the refrigerator, the suspensions were filtered over Millipore, yielding an orange compound which was re-precipitated first from benzotrifluoride $(5 \mathrm{~mL})$, and, finally, from hexafluorobenzene $(3 \times 5 \mathrm{~mL})$; this gave the title compound as a bright yellow powder. 
Yield: $140 \mathrm{mg}(70 \%)$.

${ }^{1} \mathrm{H} \mathrm{NMR}\left(500 \mathrm{MHz}, \mathrm{CDCl}_{3}\right): \delta=8.82$ (br s, $12 \mathrm{H}, \mathrm{HBC}$ ), 3.63 (br s, $\left.12 \mathrm{H}, \mathrm{CH}_{2}\right), 3.02\left(\mathrm{~m}, 12 \mathrm{H}, \mathrm{CH}_{2}\right)$.

MALDI-ICR-MS (DCTB, solvent-free preparation ${ }^{17}$ ): $\mathrm{m} / \mathrm{z}\left[\mathrm{M}^{+}\right]$ calcd for $\mathrm{C}_{90} \mathrm{H}_{36} \mathrm{~F}_{78}$ : 2598.15; found: $2598.17 ; \mathrm{m} / \mathrm{z}(\%)=2598.17$ (100), 2265.16 (15), 1932.17 (5).

UV/Vis (benzotrifluoride, $10^{-6} \mathrm{M}$ ): $\lambda_{\max }=343,359,370 \mathrm{~nm}$.

2,5,8,11,14,17-Hexakis $(5,5,6,6,7,7,8,8,9,9,10,10,10$-tridecafluorodecyl)hexabenzo[bc,ef,hi,kl,no,qr]coronene (1b)

As described for compound 1a, hexaphenylbenzene $\mathbf{2 b}(1.0 \mathrm{~g}, 0.36$ $\mathrm{mmol}$ ) was converted into the title compound in the presence of $\mathrm{CH}_{2} \mathrm{Cl}_{2}(50 \mathrm{~mL})$ and $\mathrm{FeCl}_{3}(1.17 \mathrm{~g}, 7.19 \mathrm{mmol})$ in $\mathrm{MeNO}_{2}(20 \mathrm{~mL})$.

Yield: $697 \mathrm{mg}(70 \%)$.

${ }^{1} \mathrm{H}$ NMR (500 MHz, $\left.\mathrm{CDCl}_{3}\right): \delta=9.06(\mathrm{~s}, 12 \mathrm{H}, \mathrm{HBC}), 3.57(\mathrm{t}$, $\left.J=7.8 \mathrm{~Hz}, 12 \mathrm{H}, \mathrm{CH}_{2}\right), 2.59-2.63\left(\mathrm{~m}, 24 \mathrm{H}, \mathrm{CH}_{2}\right), 2.30$ (q, ${ }^{3} J_{\mathrm{HH}}=7.8 \mathrm{~Hz}, 12 \mathrm{H}, \mathrm{CH}_{2}$ ).

MALDI-ICR-MS (DCTB, solvent-free preparation): $\mathrm{m} / z$ [ $\left.\mathrm{M}^{+}\right]$calcd for $\mathrm{C}_{102} \mathrm{H}_{60} \mathrm{~F}_{78}$ : 2766.34; found: $2766.33 ; \mathrm{m} / \mathrm{z}(\%)=2766.33(91)$, 1906.34 (20), 1644.28 (10), 1384.27 (5).

$\mathrm{UV} / \mathrm{V}$ is (benzotrifluoride, $10^{-6} \mathrm{M}$ ): $\lambda_{\max }=343,359,370 \mathrm{~nm}$.

2,5,8,11,14,17-Hexakis[6,6,7,7,8,8,9,9,10,10,11,11,11-tridecafluoro-4-(2,2,3,3,4,4,5,5,6,6,7,7,7-tridecafluoroheptyl)undecyl]hexabenzo[bc,ef,hi,kl,no,qr]coronene (1c)

As described for compound 1a, hexaphenylbenzene $2 \mathbf{c}(1.0 \mathrm{~g}, 0.21$ $\mathrm{mmol}$ ) was converted into the title compound in the presence of $\mathrm{CH}_{2} \mathrm{Cl}_{2}(80 \mathrm{~mL})$ and $\mathrm{FeCl}_{3}(0.6 \mathrm{~g}, 3.7 \mathrm{mmol})$ in $\mathrm{MeNO}_{2}(8 \mathrm{~mL})$.

Yield: $667 \mathrm{mg}(67 \%)$.

${ }^{1} \mathrm{H}$ NMR $\left(360 \mathrm{MHz}, \mathrm{CDCl}_{3}\right.$ ): $\delta=9.23$ (s, $12 \mathrm{H}, \mathrm{HBC}$ ), 2.27-2,62 (br m, $66 \mathrm{H}$ ).

MALDI-ICR-MS (DCTB, solvent-free preparation): $\mathrm{m} / z$ [M $\left.\mathrm{M}^{+}\right](\%)$ calcd for $\mathrm{C}_{150} \mathrm{H}_{78} \mathrm{~F}_{156}$ : 4842.36; found: 4842.39 (58).

UV/Vis (benzotrifluoride, $10^{-6} \mathrm{M}$ ): $\lambda_{\max }=344,361,390 \mathrm{~nm}$.

\section{References}

(1) (a) Clar, E. Polycyclic Hydrocarbons, Vol. I/II; Academic Press: New York, 1964. (b) Harvey, R. G. Polycyclic Aromatic Hydrocarbons; Wiley-VCH: New York, 1997.

(2) Wu, J.; Watson, M. D.; Zhang, L.; Wang, Z.; Müllen, K. J. Am. Chem. Soc. 2004, 126, 177.

(3) Kastler, M.; Pisula, W.; Wasserfallen, D.; Pakula, T.; Müllen, K. J. Am. Chem. Soc. 2005, 127, 4286.

(4) Spraul, K. B.; Suresh, S.; Glaser, S.; Perahia, D.; Ballato, J.; Smith, D. W. J. Am. Chem. Soc. 2004, 126, 12772.

(5) Alameddine, B.; Aebischer, O. F.; Amrein, W.; Donnio, B.; Deschenaux, R.; Guillon, D.; Savary, C.; Scanu, D.; Scheidegger, O.; Jenny, T. A. Chem. Mater. 2005, 17, 4798.

(6) Fechtenkötter, A.; Saalwächter, K.; Harbison, M. A.; Müllen, K.; Spiess, H. W. Angew. Chem. Int. Ed. 1999, 38, 3039.

(7) Vollhardt, K. P. C. Acc. Chem. Res. 1977, 10, 1.

(8) Kovacic, P.; Jones, M. B. Chem. Rev. 1987, 87, 357.

(9) Fechtenkötter, A.; Tchebotareva, N.; Watson, M.; Müllen, K. Tetrahedron 2001, 57, 3769.

(10) Mio, M. J.; Kopel, L. C.; Braun, J. B.; Gadzikwa, T. L.; Hull, K. L.; Brisbois, R. G.; Markworth, C. J.; Grieco, P. A. Org. Lett. 2002, 4, 3199.

(11) Darses, S.; Pucheault, M.; Genêt, J.-P. Eur. J. Org. Chem. 2001, 1121.

(12) Brace, N. O. J. Fluorine Chem. 1999, 93, 1.

(13) Yang, S. H.; Li, C. S.; Cheng, C. H. J. Org. Chem. 1987, 52, 691.

(14) Gambaretto, G.; Conte, L.; Fornasieri, G.; Zarantonello, C.; Tonei, D.; Sassi, A.; Bertani, R. J. Fluorine Chem. 2003, 121, 57.

(15) Bader, A.; Gladbach, B.; Arlt, D. US Patent 5,185,454, 1993.

(16) Pangborn, A. B.; Giardello, M. A.; Grubbs, R. H.; Rosen, R. K.; Timmers, F. J. Organometallics 1996, 15, 1518.

(17) Trimpin, S.; Rouhanipour, A.; Az, R.; Räder, H. J.; Müllen, K. Rapid Commun. Mass Spectrom. 2001, 15, 1364. 